\title{
Antibody responses within two leading Plasmodium vivax vaccine candidate antigens in three geographically diverse malaria-endemic regions of India
}

\author{
Sonal Kale ${ }^{1,2}$, Chander P. Yadav ${ }^{1}$, Pavitra N. Rao ${ }^{3}$, Sneh Shalini ${ }^{1}$, Alex Eapen ${ }^{4}$, Harish C. Srivasatava ${ }^{5}$,
} Surya K. Sharma ${ }^{6}$, Veena Pande ${ }^{2}$, Jane M. Carlton ${ }^{3}$, Om P. Singh ${ }^{{ }^{*}}$ and Prashant K. Mallick ${ }^{1 *}$ (D)

\begin{abstract}
Background: Identifying highly immunogenic blood stage antigens which can work as target for naturally acquired antibodies in different eco-epidemiological settings is an important step for designing malaria vaccine. Blood stage proteins of Plasmodium vivax, apical membrane antigen-1 (PvAMA-1) and $19 \mathrm{kDa}$ fragment of merozoite surface protein (PvMSP-1 ${ }_{19}$ ) are such promising vaccine candidate antigens. This study determined the naturally-acquired antibody response to PvAMA-1 and PvMSP- ${ }_{19}$ antigens in individuals living in three geographically diverse malaria endemic regions of India.
\end{abstract}

Methods: A total of 234 blood samples were collected from individuals living in three different eco-epidemiological settings, Chennai, Nadiad, and Rourkela of India. Indirect ELISA was performed to measure human IgG antibodies against recombinant PVAMA-1 and PVMSP- $1_{19}$ antigens. The difference in seroprevalence and factors associated with antibody responses at each site was statistically analysed.

Results: The overall seroprevalence was $40.6 \%$ for PvAMA-1 and $62.4 \%$ for PVMSP- ${ }_{19}$. Seroprevalence to PvAMA-1 was higher in Chennai (47\%) followed by Nadiad (46.7\%) and Rourkela (27.6\%). For PVMSP-1 ${ }_{19}$, seroprevalence was higher in Chennai (80.3\%) as compared to Nadiad (53.3\%) and Rourkela (57.9\%). Seroprevalence for both the antigens were found to be higher in Chennai where P. vivax is the dominant malaria species. In addition, heterogeneous antibody response was observed for PVAMA-1 and PVMSP- ${ }_{19}$ antigens at each of the study sites. Two factors, age and malaria positivity were significantly associated with seropositivity for both the antigens PVAMA-1 and PvMSP-1 19 .

Conclusion: These data suggest that natural acquired antibody response is higher for PVMSP- $1_{19}$ antigen as compared to PVAMA-1 antigen in individuals living in three geographically diverse malaria endemic regions in India. PVMSP-1 ${ }_{19}$ appears to be highly immunogenic in Indian population and has great potential as a malaria vaccine candidate. The differences in immune response against vaccine candidate antigens in different endemic settings should be taken into account for development of asexual stage based P. vivax malaria vaccine, which in turn can enhance malaria control efforts.

Keywords: Plasmodium vivax, PvAMA-1, PvMSP-1 ${ }_{19}$, Antibody response, Seroprevalence, India

\footnotetext{
*Correspondence: singh@mrcindia.org; pkmmrc@gmail.com

${ }^{1}$ ICMR-National Institute of Malaria Research, Sector 8, Dwarka, New

Delhi, India
}

Full list of author information is available at the end of the article 


\section{Background}

Plasmodium vivax is the most widespread human malaria parasite and a major contributor to the malaria burden outside Africa, accounting for approximately 100 million cases each year [1]. In India, the total number of confirmed malaria cases and death has been decreased in the past, but still it accounts for $52 \%$ of deaths outside of the World Health Organization (WHO) African Region [2]. As India has planned to eliminate malaria by 2030 [3], there is a need to strengthen malaria control strategies to achieve this goal. An effective malaria vaccine, which can work in diverse malaria endemic regions and provide protection against the parasite, will greatly decrease the burden of disease.

The blood stage antigens, primary target of natural acquired immunity, responsible for malaria symptoms and pathology are the main target for the malaria vaccine development [4]. To block RBC invasion and achieve blood stage growth inhibition, antigens involved in this process needs to be targeted [4]. Two of the erythrocytic stage surface proteins of Plasmodium spp. named merozoite surface protein- $1_{19}$ and apical membrane antigens-1 are the most promising candidates for malaria vaccine development due to the protective immune response against these parasite within the human and mammalian host $[5,6]$. Both are important for merozoite invasion in RBC, highly immunogenic, can induce antibody in humans and contribute towards protective immunity $[7,8]$.

AMA-1 and MSP-1 19 are well-characterized malaria vaccine candidates in Plasmodium falciparum and Plasmodium vivax $[9,10]$. The $\mathrm{C}$ terminal $19 \mathrm{kDa}$ region of MSP-1 remains on the surface of merozoites and initially plays role during adhesion of merozoites to RBCs [11-14]. The AMA-1 is an integral membrane protein expressed by merozoites and sporozoites [15]. This surface protein becomes crucial at the time of erythrocyte invasion as it is involved in the reorientation of merozoites [16]. Furthermore, during invasion AMA-1 binds to rhoptry neck protein (RON2) and forms the junction complex [16]. Several studies have reported that antibody against these antigens can inhibit the erythrocyte invasion by merozoites and it is associated with a decreased risk of malaria [6, 12, 17]. Individuals living in malaria endemic regions develop an effective immune response against the parasite and are less susceptible to malaria infection [18]. Moreover, population living in such endemic areas have been shown to possess anti-AMA-1 and anti-MSP- $1_{19}$ antibodies, which increases with age [19-21]. Many in vitro and animal model studies have also shown that such antibodies can reduce parasite multiplication and protect from lethal infection [22-25].
The unique geographic position and diverse climate of India make it perfect for malaria transmission and presents challenges towards malaria control and elimination. An understanding of the host immune response, acquisition and maintenance of the antimalarial antibody to $P$. vivax vaccine candidate antigens in people living in malaria endemic areas is crucial for improving prospects on successful malaria vaccine development [26, 27]. Here, the antibody responses to recombinant $P$. vivax apical membrane antigen 1 (PvAMA-1) and merozoite surface antigen-1 19 (PvMSP-1 19 ) were investigated in individuals living at three geographically diverse malaria endemic regions of India. The immune status of the residents living in diverse $P$. vivax transmission area and factors associated with it has not been reported from India. Results of this study would be a support to evaluate the malaria vaccine development and elimination programme in India.

\section{Methods \\ Study sites}

The details of three field sites of the Center for the Study of Complex Malaria in India (CSCMi) i.e., Nadiad (Gujarat), Chennai (Tamil Nadu) and Rourkela (Odisha) have been described previously [28, 29]. These selected study sites represented different eco-epidemiological conditions, malaria vector system, transmission rates and relative prevalence of $P$. vivax and $P$. falciparum. Briefly, Chennai is the capital city of Tamil Nadu state (Fig. 1). Malaria transmission in Chennai is perennial due to humid and hot climate and malaria cases increases between July and October. Anopheles stephensi is the main malaria vector in Chennai and $P$. vivax is the dominant malaria species [30,31]. In Chennai, annual parasite incidence (API, number of malaria cases per thousand population) was 2.34 in 2012 which reduced to 1.79 in 2013 [3]. Samples were collected from individuals enrolled at the Besant Nagar Malaria Clinic or in crosssectional surveys conducted in few slums, urban dwellings and a large coastal community near the Besant Nagar area. Nadiad town is located in Kheda district of Gujarat state. Here, P. vivax and P. falciparum malaria occur throughout the year with a slightly higher prevalence of $P$. vivax. Nadiad has semi-arid and sub-tropical climate. In Nadiad, Anopheles culicifacies is the main malaria vector and API 2.5 observed in 2010 [3, 28] Samples were collected from individuals enrolled at a malaria clinic in Nadiad Civil Hospital and in cross-sectional survey conducted at nearby rural area of Nadiad town. Rourkela, is located in Sundargarh district of Odisha state and has a tropical wet and dry climate. Anopheles culicifacies and Anopheles fluviatilis are main malaria vectors with $P$. 


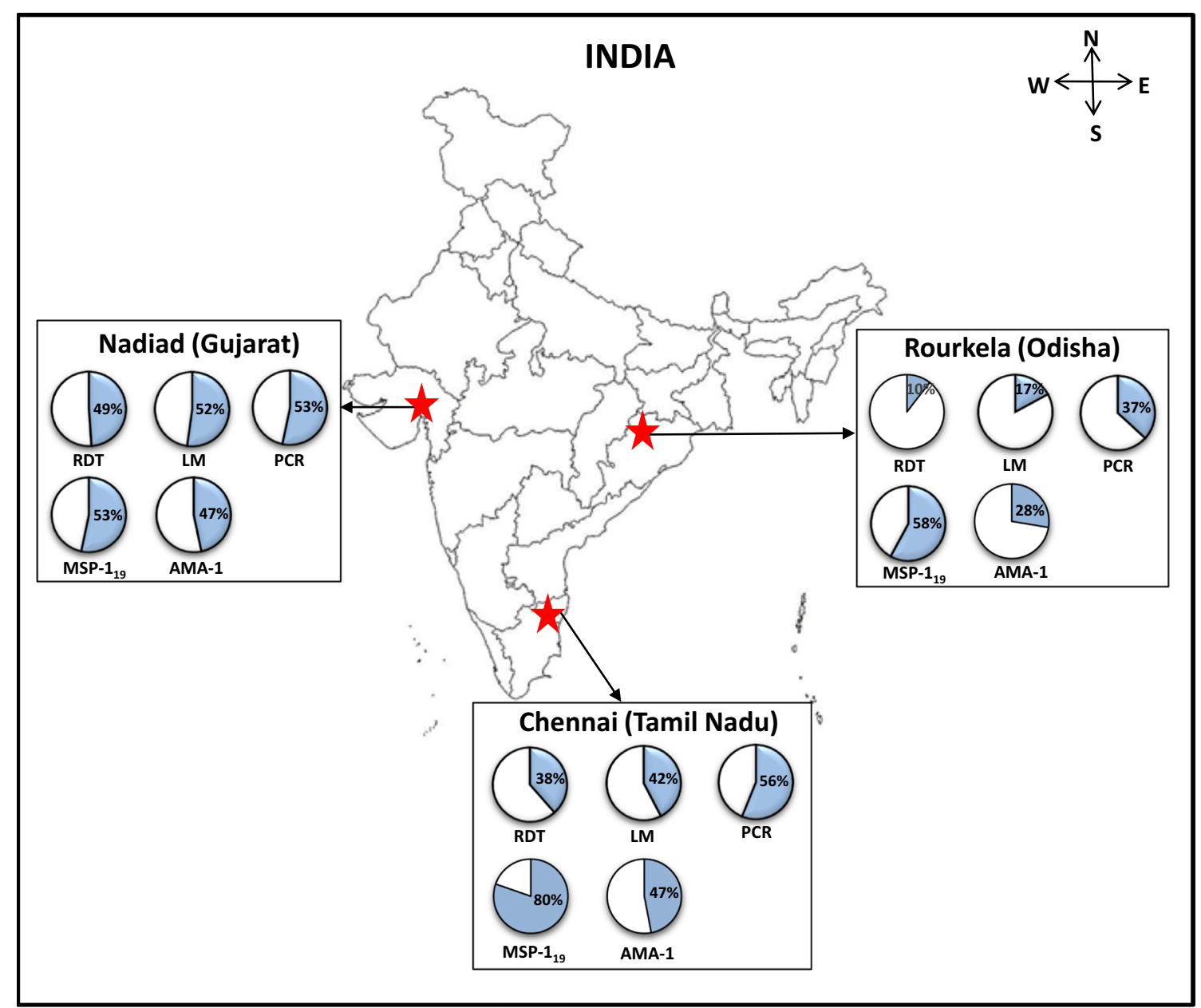

Fig. 1 Map showing three study sites and the proportion of Plasmodium vivax infection evaluated by different methods. The pie graph represents the proportion (\%) of P. vivax infection or seropositivity (\%) for PVMSP- ${ }_{19}$ and PvAMA-1 antigens. RDT is the rapid diagnostic test, LM is light microscopy and PCR is diagnostic PCR performed for species identification

falciparum as a dominant malaria parasite species. It has highest API 7.57 among three selected sites in 2010 [3, 28]. Samples were collected from individuals enrolled at health clinic and from cross-sectional surveys conducted in rural areas of Rourkela.

\section{Sample collection and processing}

Approximately 3 to $5 \mathrm{~mL}$ of blood was collected in EDTA vacutainers (Thermo Fisher, Massachusetts, USA) from each individual in cross-sectional surveys $(\mathrm{CSS}, \mathrm{N}=98)$ and clinic $(\mathrm{CL}, \mathrm{N}=136)$ at three field sites Chennai $(\mathrm{CSS}=11, \mathrm{CL}=55)$, Nadiad $(\mathrm{CSS}=32, \mathrm{CL}=60)$ and Rourkela $(C S S=55, C L=21)$ during January 2013-May 2015. Plasma samples from 234 individuals (aged 1-70) were used for this study. Blood samples were centrifuged at $1500 \times g$ for $15 \mathrm{~min}$ at room temperature and plasma was removed and stored at $-80^{\circ} \mathrm{C}$. DNA extraction was performed using red blood cells by QiAamp DNA blood mini kits (Qiagen Inc., Valencia, CA). Individuals were enquired about their history of malaria in last 12 months, fever within last 2 weeks, do they had antimalarial drugs in last 2 weeks or taking any fever suppressants now, if they use bed nets, their age, gender were recorded.

Malaria infection was diagnosed by three test, rapid diagnostic test (RDT), blood smear microscopy, and polymerase chain reaction (PCR) for all samples collected in the cross sectional surveys and clinic studies. Malaria infection was determined by bivalent RDT (FalciVax, Zephyr Biomedicals, India) by finger prick blood at the time of enrollment, followed by microscopic examination of thick blood smear, stained with Giemsa by microscopy using a $100 \times$ oil immersion objective. Parasites were counted on the thick smear against 200-500 leucocytes (WBC) and expressed as parasites per microlitre 
of blood, using the WBC count if known, or assuming 8000 WBCs per microlitre of blood. A semi nested species specific PCR assay was used as described earlier [32] for molecular detection of $P$. vivax and/or P. falciparum. Infection of Plasmodium spp. was reconfirmed by diagnostic PCR at National Institute of Malaria Research Delhi and only $P$. vivax positive and malaria negative samples were selected for the study.

\section{Enzyme-linked immunosorbent assay (ELISA)}

The total IgG antibody response against the $P$. vivax recombinant apical membrane antigen-1 (PvAMA1) and the $19 \mathrm{kDa}$ fragment of recombinant merozoite surface protein (PvMSP-1 $1_{19}$ ) synthesized at London School of Hygiene and Tropical Medicine, London, UK as described previously [16,33-35] were detected by an indirect ELISA as described previously [33, 36]. Briefly, recombinant PvAMA-1 (SalI strain) and PvMSP-1 19 (Belem strain) antigens were diluted in coating buffer (0.15 M sodium carbonate, $0.034 \mathrm{M}$ sodium bicarbonate, $\mathrm{pH}$ 9.6) to $0.5 \mu \mathrm{g} / \mathrm{mL}$ concentration and $50 \mu \mathrm{L}$ of each diluted antigen were coated on 96 well ELISA plate (Immulon 4 HBX, Thermo Scientific). Plates were incubated overnight at $4{ }^{\circ} \mathrm{C}$. After incubation, ELISA plates were washed with washing buffer $(1 \times$ PBS, $0.05 \%$ Tween 20) $(\mathrm{PBS} / \mathrm{T})$ and blocked with $1 \%$ skimmed milk solution for $3 \mathrm{~h}$. After washing plates with PBS/T solution, serum samples were added in duplicates at a final dilution of 1:1000 for PvMSP- $1_{19}$ and 1:2000 for PvAMA-1 to each plate together with serial dilution of positive control (pooled hyper immune serum of 20 adults from Sepik, Papua New Guinea) and negative control (pooled serum samples from healthy individuals from USA who never experienced malaria). These plates were incubated overnight at $4{ }^{\circ} \mathrm{C}$ and washed with PBS/T solution. $50 \mu \mathrm{L}$ of horseradish peroxidase-conjugated rabbit anti-human IgG (DAKO), diluted $1 / 5000$ in $\mathrm{PBS} / \mathrm{T}$, were added in each wells, incubated at room temperature for $3 \mathrm{~h}$ and washed again with PBS/T solution. Ortho-phenylenediamine substrate (Sigma) was added $100 \mu \mathrm{L}$ in each well and left in dark for 10-15 $\mathrm{min}$ at room temperature. $25 \mu \mathrm{L}$ of stop solution $\left(2 \mathrm{M} \mathrm{H}_{2} \mathrm{SO}_{4}\right)$ was added in each well to stop the reaction and optical density (OD) at $492 \mathrm{~nm}$ was recorded using ELISA reader (NanoQuant, TECAN).

\section{Statistical analysis}

Optical density (OD) values recorded in duplicate were averaged and normalized against blank wells values to adjust the background reactivity. To calculate seropositivity a separate cut-off was generated for each antigen. A plasma sample was considered seropositive when absorbance was higher than the mean OD plus twice of standard deviations (SD) of negative control (pooled serum samples from unexposed individuals in the USA). Titre values were calculated by plotting a titration curve, using the normalized OD values of serially diluted positive control in Microsoft-Excel as described previously [36]. All descriptive data (categorical variables) were expressed in number $(\mathrm{N})$ and percentage (\%). Seroprevalence was calculated as a percentage for participants who was seropositive either for PvAMA-1 or PvMSP- $1_{19}$ separately and expressed as a proportion. 95\% confidence interval (CI) was estimated using binomial distribution. All continuous normally distributed variables were expressed as mean \pm SD while non-normally distributed variable was expressed as Median (P25 to P75). Normality assumption was checked using QQ plot, histogram and KolmogorovSmirnov test. Association between categorical variables was assessed using Chi-Square/Fisher exact test. In order to know the factors which may affect the seropositivity rate of PvAMA-1 and PvMSP-1 ${ }_{19}$, association of various factors with seropositivity to PvAMA- 1 and PvMSP- $1_{19}$ was assessed using logistic regression, separately for each of the antigens. Followed by bi-variable analysis, a multivariable analysis was done using step-wise multivariable logistic regression. P-value of less than 0.05 was considered as significant and all statistical analysis was done using statistical software R 3.4 and Stata 15.0.

\section{Results}

\section{Baseline characteristics}

A total of 234 individuals were recruited from the three geographically diverse malaria endemic regions of India namely Chennai $(n=66)$, Nadiad $(n=92)$ and Rourkela $(\mathrm{n}=76)$ in January 2013-May 2015. Age of the study participants ranged between 25 and 48 years (median $=32$ years) in Chennai, $15.5-41$ years (median $=29$ years) in Nadiad and 11-35 years (median $=23$ years) in Rourkela. The majority of participants were adults at each site. More than $60 \%$ study participants were male $(n=146)$. Overall, the bed net users were $70.51 \%$. The bed net users were higher in Chennai (96.97\%) and Nadiad (90.22\%) while in Rourkela it was only $23.68 \%$. Total $25 \%$ participants were having fever within last 2 weeks and were taking fever suppressant at the time of enrollment. Only 3.4\% participants had taken anti-malarial drugs in last 2 weeks. In Rourkela, $25 \%$ of the study population recalled (by memory) that they may had one or more than one attack of malaria infection (either $P$. vivax or P. falciparum) in past 12 months, while in Chennai and Nadiad the percentage of a previous history of malaria was $25.76 \%$ and $5.43 \%$ respectively. Previous malaria exposure was higher and comparable in Rourkela and Chennai population as compare to Nadiad. More details of the study participants at each site are shown in Table 1. 
Table 1 Baseline information

\begin{tabular}{|c|c|c|c|c|}
\hline \multirow[t]{2}{*}{ Characteristic } & \multicolumn{3}{|l|}{ Study site } & \multirow[t]{2}{*}{ Overall $(\mathrm{N}=234)$} \\
\hline & Chennai $(N=66)$ & Nadiad $(\mathrm{N}=92)$ & Rourkela $(\mathrm{N}=76)$ & \\
\hline Age (years) & $32(25-48)$ & $29(15.5-41)$ & $23(11-35)$ & $29(17-40)$ \\
\hline Gender, male & $41(62.1)$ & $60(65.2)$ & $45(59.2)$ & $146(62.4)$ \\
\hline Bed net use & $64(97)$ & $83(90.2)$ & $18(23.7)$ & $165(70.5)$ \\
\hline Fever within last 2 weeks & $20(32.8)$ & $29(31.5)$ & $10(13.2)$ & $59(25.8)$ \\
\hline Fever suppressant & $54(81.8)$ & $29(31.5)$ & $19(25.0)$ & $102(43.6)$ \\
\hline $\begin{array}{l}\text { Taken anti-malarial drugs in last } \\
2 \text { weeks }\end{array}$ & $1(1.6)$ & $4(4.3)$ & $3(3.9)$ & $8(3.4)$ \\
\hline Malaria in last 12 months & $17(25.8)$ & $5(5.4)$ & $19(25.0)$ & $41(17.5)$ \\
\hline RDT positive & $25(37.9)$ & $45(48.9)$ & $8(10.5)$ & $78(33.3)$ \\
\hline Microscopy positive & $28(42.4)$ & $48(52.2)$ & $13(17.1)$ & $89(38.0)$ \\
\hline PCR positive & $37(56.1)$ & $49(53.3)$ & $28(36.8)$ & $114(48.7)$ \\
\hline \multicolumn{5}{|l|}{ Malaria cases } \\
\hline Asymptomatic & $28(75.7)$ & $27(55.1)$ & $25(89.3)$ & $80(70.2)$ \\
\hline Symptomatic & $9(24.3)$ & $22(44.9)$ & $3(10.7)$ & $34(29.8)$ \\
\hline \multicolumn{5}{|l|}{ Parasite count } \\
\hline Low $(\leq 5000)$ & $25(86.2)$ & 35 (72.9) & $10(58.8)$ & $70(74.5)$ \\
\hline High (> 5000) & $4(13.8)$ & $13(27.1)$ & 7 (41.2) & $24(25.5)$ \\
\hline
\end{tabular}

Number are expressed in n (\%) and median (P25 to P75)

Overall P. vivax prevalence was $33.3 \%, 38.0 \%$ and $48.7 \%$ detected by RDT, microscopy and PCR methods, respectively. A total of 114 individuals were $P$. vivax malaria positive and 120 individuals were malaria negative as diagnosed by PCR. Malaria positivity as detected by PCR varied among settings, i.e., 37 (56.06\%) in Chennai, 49 (53.26\%) in Nadiad and 28 (36.84\%) in Rourkela (Fig. 1). Irrespective of diagnostic method, higher $P$. vivax malaria prevalence was observed in Chennai and Nadiad than Rourkela.

Among the studied population, $70(74.5 \%)$ subjects were asymptomatic (body temperature $<37.5{ }^{\circ} \mathrm{C}$ and PCR positive) and $24(25.5 \%)$ subjects were symptomatic (body temperature $>37.5^{\circ} \mathrm{C}$ and PCR positive). The number of asymptomatic subjects was higher at each site as compared to the number of symptomatic subjects. Overall $74.5 \%$ population were having low parasitaemia ( $\leq 5000$, asexual parasites) and only $25.5 \%$ population were having high parasitaemia ( $>5000$, asexual parasites) (Table 1).

\section{Natural acquired antibody response against malaria antigens at three study sites in India PvAMA-1 antibodies}

The antibody response whether evaluated as OD level, antibody titre or seroprevalence increased with increasing $P$. vivax prevalence. The overall PvAMA-1 seroprevalence was $40.6 \%$ (95\% CI 34.4-47.1), which was higher in Chennai (47\%, 95\% CI 35.0-59.3) and Nadiad (46.7\% 95\%
CI 36.6-57.1) than Rourkela (27.6\%, 95\% CI 18.6-39). Seroprevalence between male and female was varying across three sites, higher seroprevalence was observed for males $(56.1 \%)$ than females $(32.0 \%)$ in Chennai. While in other two sites, higher seroprevalence was observed for females (Nadiad 56.2\%, Rourkela 35.5\%) than males (Nadiad 41.7\%, Rourkela 22.2\%). For Nadiad and Rourkela, higher seroprevalence was observed in adults ( $\geq 15$ years) (Nadiad $50 \%$, Rourkela 35.3\%) than children (<15 years) (Nadiad 35\%, Rourkela 12\%) while in Chennai we had only adult participant whose seroprevalence was $47.0 \%$. PvAMA-1 seroprevalence was higher in malaria positive (PCR positive) participants at each site as compared to malaria negative (PCR negative). Seroprevalence was higher in symptomatic individuals in Nadiad (81.8\%), and Rourkela (66.7\%) whereas in Chennai seroprevalence was higher in asymptomatic individuals (64.3\%) as compared to symptomatic (55.6\%). Sero-response was comparable between those who have high parasitaemia and those have low parasitaemia, and pattern was similar across sites (Table 2). Antibody titre for PvAMA-1 was higher in Chennai (mean $=248.34$ ), followed by Nadiad $($ mean $=111.74)$ and Rourkela $($ mean $=67.11)$. Antibody response was higher in Chennai and Nadiad population as compared to Rourkela (Figs. 2 and 3).

\section{PvMSP-1 ${ }_{19}$ antibodies}

The overall seroprevalence for PvMSP- $1_{19}$ was $62.4 \%$ (95\% CI 56-68.4) which was higher than PvAMA-1 
Table 2 Antibody response against PvAMA-1 at three sites in India

\begin{tabular}{|c|c|c|c|c|c|c|c|c|}
\hline \multirow[t]{2}{*}{ Seroprevalence } & \multicolumn{2}{|l|}{ Chennai } & \multicolumn{2}{|l|}{ Nadiad } & \multicolumn{2}{|l|}{ Rourkela } & \multicolumn{2}{|l|}{ Total } \\
\hline & n/N (\%) & $95 \% \mathrm{Cl}$ & n/N (\%) & $95 \% \mathrm{Cl}$ & n/N (\%) & $95 \% \mathrm{Cl}$ & n/N (\%) & $95 \% \mathrm{Cl}$ \\
\hline Overall & $31 / 66$ (47.0) & $35.0-59.3$ & 43/92 (46.7) & $36.6-57.1$ & 21/76 (27.6) & $18.6-39.0$ & 95/234 (40.6) & $34.4-47.1$ \\
\hline \multicolumn{9}{|l|}{ Gender } \\
\hline Male & 23/41 (56.1) & $40.2-70.9$ & 25/60 (41.7) & $29.6-54.8$ & 10/45 (22.2) & $12.1-37.2$ & $58 / 146(39.7)$ & $32.0-48.0$ \\
\hline Female & 8/25 (32.0) & $16.0-53.7$ & 18/32 (56.2) & $38.1-72.9$ & 11/31 (35.5) & $20.1-54.5$ & 37/88 (42.0) & $32.0-52.7$ \\
\hline \multicolumn{9}{|l|}{ Age (years) } \\
\hline$<15$ & 0 & 0 & $7 / 20$ (35.0) & $16.4-59.6$ & $3 / 25(12.0)$ & $03.6-33.3$ & 10/45 (22.2) & $12.1-37.2$ \\
\hline$\geq 15$ & $31 / 66$ (47.0) & $35.0-59.3$ & $36 / 72(50.0)$ & $38.4-61.6$ & 18/51 (35.3) & $23.1-49.7$ & $85 / 189$ (45.0) & $38.0-52.2$ \\
\hline \multicolumn{9}{|l|}{ PCR } \\
\hline Positive & 23/37 (62.2) & $45.0-76.7$ & 39/49 (79.6) & $65.5-88.9$ & 13/28 (46.4) & $28.2-65.7$ & 75/114 (65.8) & $56.5-74.0$ \\
\hline Negative & 8/29 (27.6) & $13.1-47.5$ & 4/43 (9.3) & $03.4-23.0$ & 8/48 (16.7) & $08.3-30.5$ & 20/120 (16.7) & $10.9-24.5$ \\
\hline \multicolumn{9}{|l|}{ Malaria } \\
\hline Asymptomatic & 18/28 (64.3) & $44.1-80.4$ & 21/27 (77.8) & $57.0-90.2$ & $11 / 25(44.0)$ & $25.2-64.7$ & $50 / 80(62.5)$ & $51.2-72.6$ \\
\hline Symptomatic & $5 / 9(55.5)$ & $19.5-86.6$ & 18/22 (81.8) & $58.1-93.6$ & $2 / 3(66.7)$ & $0.3-99.9$ & 25/34 (73.5) & $55.4-86.1$ \\
\hline \multicolumn{9}{|l|}{ Parasitaemia } \\
\hline Low & 19/25 (76.0) & $54.1-89.5$ & 28/35 (80.0) & $62.6-90.5$ & $5 / 10(50.0)$ & 18.1-81.9 & $52 / 70(74.3)$ & $62.5-83.3$ \\
\hline High & $3 / 4(75.0)$ & $4.1-99.5$ & 11/13 (84.6) & 49.0-96.9 & 4/7 (57.1) & $15.0-90.9$ & 18/24 (75.0) & $52.5-89.0$ \\
\hline
\end{tabular}

$\mathrm{Cl}$ confidence interval
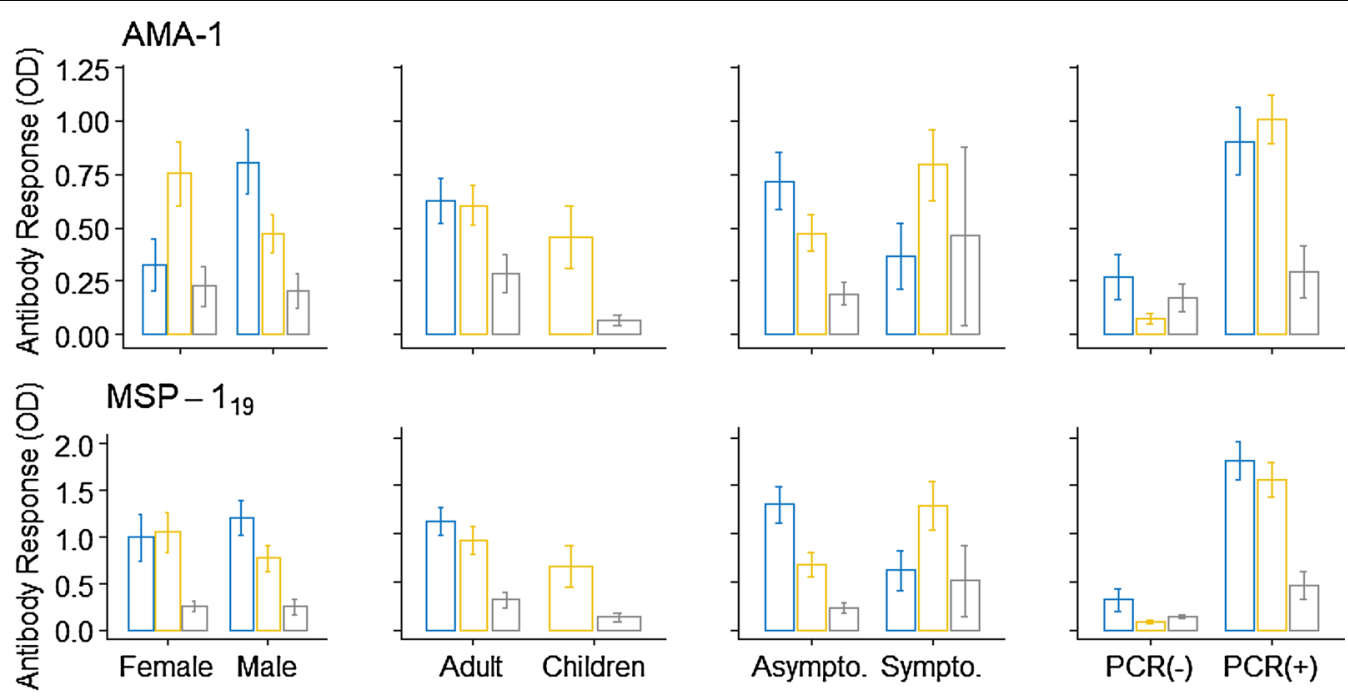

site $\square$ Chennai $\square$ Nadiad $\square$ Rourkela

Fig. 2 Antibody response against PVAMA-1 and PvMSP-1 19 antigens as determined by optical density (OD) by ELISA, shown in different categories of gender, age, symptoms, and malaria -positivity by each sites using error bar plot. Here bar represents mean and error bar over it represents standard error

seroprevalence (40.6\%, 95\% CI 34.4-47.1) across three study sites. PvMSP-1 $1_{19}$ seroprevalence was higher in Chennai (80.3\%), compared to Nadiad $(53.3 \%)$ and Rourkela (57.9\%) (Table 3). Higher seroprevalence was observed in females in Nadiad (62.5\%) and Rourkela $(61.3 \%)$ while in Chennai higher seroprevalence was observed in males (82.9\%) as compared to females (76.0\%). Between two age groups higher seroprevalence was found in adults at all three study sites (Fig. 2). Seroprevalence to PvMSP-1 19 antigen in $P$. vivax infected (PCR confirmed) individuals was higher in 


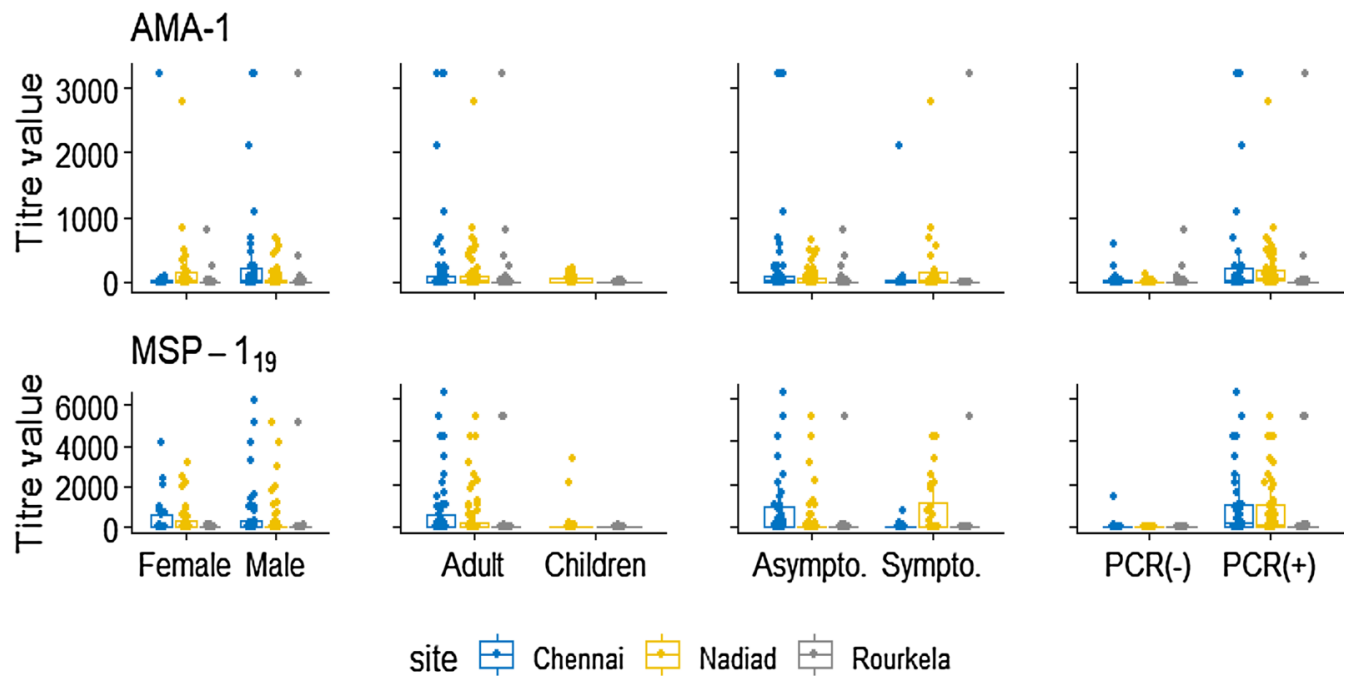

Fig. 3 Antibody level against PVAMA-1 and PvMSP- $1_{19}$ antigens as determined by titer value (IQR, interquartile range 25th-75th percentile) presented specific to gender, age-categories, symptoms and malaria positivity by each sites using Box-Whiskers plot

Table 3 Antibody response against PvMSP-1 ${ }_{19}$ at three sites in India

\begin{tabular}{|c|c|c|c|c|c|c|c|c|}
\hline \multirow[t]{2}{*}{ Seroprevalence } & \multicolumn{2}{|l|}{ Chennai } & \multicolumn{2}{|l|}{ Nadiad } & \multicolumn{2}{|l|}{ Rourkela } & \multicolumn{2}{|l|}{ Total } \\
\hline & n/N (\%) & $95 \% \mathrm{Cl}$ & n/N (\%) & $95 \% \mathrm{Cl}$ & n/N (\%) & $95 \% \mathrm{Cl}$ & $\mathrm{n} / \mathrm{N}(\%)$ & $95 \% \mathrm{Cl}$ \\
\hline Overall & $53 / 66(80.3)$ & $68.6-88.4$ & 49/92 (53.3) & $42.9-63.4$ & 44/76 (57.9) & $46.3-68.7$ & $146 / 234(62.4)$ & $56.0-68.4$ \\
\hline \multicolumn{9}{|l|}{ Gender } \\
\hline Male & 34/41 (82.9) & $67.5-91.9$ & 29/60 (48.3) & $35.7-61.2$ & 25/45 (55.5) & $40.4-69.7$ & $88 / 146(60.3)$ & $52.0-68.0$ \\
\hline Female & 19/25 (76.0) & $54.1-89.5$ & $20 / 32(62.5)$ & $43.9-78.0$ & 19/31 (61.3) & $42.4-77.3$ & $58 / 88(65.9)$ & $55.2-75.2$ \\
\hline \multicolumn{9}{|l|}{ Age (years) } \\
\hline$<15$ & 0 & - & $10 / 20(50.0)$ & $27.7-72.3$ & $11 / 25(44.0)$ & $25.2-64.7$ & $21 / 45(46.7)$ & $32.2-61.7$ \\
\hline$\geq 15$ & $53 / 66(80.3)$ & $68.6-88.4$ & $39 / 72(54.2)$ & $42.4-65.5$ & $33 / 51(64.7)$ & $50.3-76.9$ & $125 / 189(66.1)$ & $59.0-72.6$ \\
\hline \multicolumn{9}{|l|}{ PCR125/189 (66.1) } \\
\hline Positive & 31/37 (83.8) & $67.4-92.8$ & 42/49 (85.7) & 72.4-93.2 & 15/28 (53.6) & $34.3-71.8$ & $88 / 114(77.2)$ & $68.5-84.1$ \\
\hline Negative & 22/29 (75.9) & $56.0-88.6$ & $7 / 43(16.3)$ & $07.7-31.1$ & 29/48 (60.4) & $45.6-73.5$ & $58 / 120(48.3)$ & $39.4-57.4$ \\
\hline \multicolumn{9}{|l|}{ Malaria } \\
\hline Asymptomatic & $24 / 28(85.7)$ & $66.0-94.9$ & 25/27 (92.6) & $72.8-98.3$ & $12 / 25(48.0)$ & $28.4-68.2$ & $61 / 80(76.2)$ & $65.5-84.4$ \\
\hline Symptomatic & $7 / 9(77.8)$ & $33.0-96.1$ & 17/22 (77.3) & $53.5-90.9$ & $3 / 3(100)$ & - & 27/34 (79.4) & $61.6-90.2$ \\
\hline \multicolumn{9}{|l|}{ Parasitaemia } \\
\hline Low & 23/25 (92) & 70.9-98.2 & $30 / 35(85.7)$ & $68.9-94.2$ & $5 / 10(50.0)$ & $18.1-81.9$ & $58 / 70(82.8)$ & 71.9-90.1 \\
\hline High & $4 / 4(100)$ & - & $12 / 13(92.3)$ & $53.1-99.2$ & $3 / 7$ (42.8) & $9.1-84.9$ & 19/24 (79.2) & $56.8-91.7$ \\
\hline
\end{tabular}

Cl confidence interval

Chennai (83.8\%) and Nadiad (85.7\%), but Rourkela showed slightly high seropositivity in malaria negative individuals $(60.2 \%)$ as compared to $P$. vivax positive individuals (53.6\%). Seroprevalence was higher in asymptomatic individuals as compared to symptomatic patients in Nadiad (92.6\%) and Chennai (85.7\%), whereas in Rourkela seroprevalence was higher in symptomatic individuals (100\%). Antibody response between high parasitaemia and low parasitaemia group was almost equal at each site (Table 3). Antibody titre for PvMSP-1 19 antigen was higher in Chennai (mean $=593.70)$ and Nadiad (mean $=461.46)$ than Rourkela (mean $=143.36)$. Overall antibody titre was higher for PvMSP-1 19 (mean = 395.44) as compared to PvAMA-1 $($ mean $=135.77)$. 


\section{Factors associated with seropositivity to PvAMA-1} and PvMSP-1 19

A total of ten factors (sex, age, malaria positivity by PCR, symptomatic and asymptomatic malaria, Parasitaemia, fever within 2 weeks, taking fever suppressants, anti-malarial drugs, malaria in last 12 months and diverse eco-epidemiological study sites) were considered as potential predictors of seropositivity for both antigens. Among these, four predictors (age, malaria positivity using PCR, fever in last 2 weeks, and study sites) were found statistical significant in bi-variate analysis for PvAMA-1. In multivariable analysis, done by step-wise logistic regression, two predictors viz. age and malaria positivity by PCR were found to significantly associated with seropositivity of PvAMA-1. Odds of being seropositive to PvAMA-1 for adult ( $\geq 15$ years) was almost four fold [OR (95\% CI) 4.12 (1.76-10.11)] higher than children (age $<15$ years). Odds of being seropositive to PvAMA-1 for malaria diagnosed person using PCR was almost 13 [OR (95\% CI) $13.02(6.52-22.50)]$ times more than the person who do not have malaria (Table 4).

Similar analysis was done for second antigen i.e. PvMSP-1 $1_{19}$. In this case, six factors (age, malaria positive by PCR, fever within 2 weeks, fever suppressant, malaria in last 12 month and study sites) out of ten factors showed association with seropositive to

Table 4 Factors associated with seropositivity to PvAMA-1

\begin{tabular}{|c|c|c|c|c|c|}
\hline \multirow[t]{2}{*}{ Factors } & \multirow[t]{2}{*}{ Seropositive, $n / N(\%)$} & \multicolumn{2}{|l|}{ Unadjusted } & \multicolumn{2}{|l|}{ Adjusted } \\
\hline & & OR $(95 \% \mathrm{Cl})$ & $P$ value & OR $(95 \% \mathrm{Cl})$ & $P$ value \\
\hline \multicolumn{6}{|l|}{ Gender } \\
\hline Male & $58 / 146(39.73)$ & Ref. & 0.726 & a & \\
\hline Female & 37/88 (42.05) & $0.91(0.53-1.55)$ & & & \\
\hline \multicolumn{6}{|l|}{ Age } \\
\hline$<15$ & 10/45 (22.22) & Ref. & 0.007 & Ref. & 0.001 \\
\hline$\geq 15$ & $85 / 189(44.97)$ & $2.86(1.34-6.11)$ & & $4.12(1.76-10.11)$ & \\
\hline \multicolumn{6}{|l|}{ PCR } \\
\hline Positive & 75/114 (65.79) & Ref. & $<0.001$ & Ref. & $<0.001$ \\
\hline Negative & $20 / 120(16.67)$ & $9.61(5.19-17.81)$ & & $13.02(6.52-25.50)$ & \\
\hline \multicolumn{6}{|l|}{ Malaria cases } \\
\hline Asymptomatic & $69 / 80(72.63)$ & Ref. & 0.119 & a & \\
\hline Symptomatic & $26 / 34(27.37)$ & $1.64(0.88-3.04)$ & & & \\
\hline \multicolumn{6}{|l|}{ Parasite count } \\
\hline Low & $52 / 70(74.29)$ & Ref. & 0.945 & a & \\
\hline High & $18 / 24(75.00)$ & $1.04(0.36-3.02)$ & & & \\
\hline \multicolumn{6}{|c|}{ Fever within last 2 week } \\
\hline No & $55 / 170(32.35)$ & Ref. & $<0.001$ & a & \\
\hline Yes & $38 / 59(64.41)$ & $3.78(2.03-7.05)$ & & & \\
\hline \multicolumn{6}{|l|}{ Fever suppressant } \\
\hline No & 47/132 (35.61) & Ref. & 0.078 & a & \\
\hline Yes & $48 / 102(47.06)$ & $1.61(0.95-2.72)$ & & & \\
\hline \multicolumn{6}{|c|}{ Taken anti-malarial drug } \\
\hline No & $90 / 224(40.18)$ & Ref. & 0.580 & a & \\
\hline Yes & $4 / 8(50.00)$ & $1.48(0.36-6.11)$ & & & \\
\hline \multicolumn{6}{|c|}{ Malaria in last 12 months } \\
\hline No & 74/193 (38.34) & Ref. & 0.130 & a & \\
\hline Yes & $21 / 41(51.22)$ & $1.69(0.85-3.32)$ & & & \\
\hline \multicolumn{6}{|l|}{ Study sites } \\
\hline Rourkela & $21 / 76(27.63)$ & Ref. & & a & \\
\hline Chennai & $31 / 66(46.97)$ & $2.31(1.15-4.65)$ & 0.018 & & \\
\hline Nadiad & 43/92 (46.74) & $2.30(1.20-4.39)$ & 0.012 & & \\
\hline
\end{tabular}

OR odds ratio

a Omitted in multivariable analysis 
PvMSP- $1_{19}$ in bi-variable analysis. In multivariable analysis four predictors (viz. age, malaria positive by PCR, fever within last 2 weeks, and malaria in last 12 months) were identified significantly associated with seropositivity of PvMSP- $1_{19}$. Odds of being seropositive to PvMSP- $1_{19}$ for adult ( $\geq 15$ years) was almost two fold [OR (95\% CI) $2.70(1.25-5.85)$ ] higher than children (age $<15$ years). Odds of being seropositive to PvMSP- $1_{19}$ for malaria diagnosed person using PCR was also almost two [OR (95\% CI) 2.44 (1.29-4.63)] times more than the person who do not have malaria. Odds of being seropositive to PvMSP- $1_{19}$ of those who experienced fever in last 2 weeks five times [OR (95\%
CI) $5.70(2.19-14.84)]$ more than those who were free from fever in last 2 weeks. Odds of being seropositive to PvMSP- $1_{19}$ of those who experienced malaria fever in last 12 months was three times [OR (95\% CI) 3.65 (1.48-9.03)] higher than those who did not experience malaria in last 12 months (Table 5).

\section{Discussion}

In India, insight to naturally acquired antibody response to leading $P$. vivax vaccine candidate antigens for development of an effective vaccine that can work in diverse regions is very limited. The present study is first attempt to investigate antibody response against $P$. vivax vaccine

Table 5 Factors associated with seropositivity to PvMSP-1 19

\begin{tabular}{|c|c|c|c|c|c|}
\hline \multirow[t]{2}{*}{ Factors } & \multirow[t]{2}{*}{ Seropositive, n/N (\%) } & \multicolumn{2}{|l|}{ Unadjusted } & \multicolumn{2}{|l|}{ Adjusted } \\
\hline & & OR $(95 \% \mathrm{Cl})$ & $P$ value & OR $(95 \% \mathrm{Cl})$ & $P$ value \\
\hline \multicolumn{6}{|l|}{ Gender } \\
\hline Male & $88 / 146(60.27)$ & Ref. & 0.389 & a & \\
\hline Female & $58 / 88(65.91)$ & $0.78(0.45-1.36)$ & & & \\
\hline \multicolumn{6}{|l|}{ Age } \\
\hline$<15$ & $21 / 45(46.67)$ & Ref. & 0.017 & Ref. & 0.011 \\
\hline$\geq 15$ & $125 / 189(66.14)$ & $2.23(1.15-4.31)$ & & $2.70(1.25-5.85)$ & \\
\hline \multicolumn{6}{|l|}{ PCR } \\
\hline Positive & $88 / 114(60.27)$ & Ref. & 0.000 & Ref. & 0.006 \\
\hline Negative & $58 / 120(39.73)$ & $3.62(2.05-6.37)$ & & $2.44(1.29-4.63)$ & \\
\hline \multicolumn{6}{|l|}{ Malaria cases } \\
\hline Asymptomatic & $61 / 80(76.2)$ & Ref. & 0.713 & a & - \\
\hline Symptomatic & $27 / 34(79.4)$ & $1.20(0.45-3.19)$ & & & \\
\hline \multicolumn{6}{|l|}{ Parasite count } \\
\hline Low & $58 / 70(82.86)$ & Ref. & 0.686 & & \\
\hline High & $19 / 24(79.17)$ & $0.79(0.24-2.52)$ & & & \\
\hline \multicolumn{6}{|c|}{ Fever within last 2 week } \\
\hline No & $89 / 170(52.35)$ & Ref. & 0.000 & Ref. & $<0.001$ \\
\hline Yes & $53 / 59(89.83)$ & $8.04(3.28-19.70)$ & & $5.70(2.19-14.84)$ & \\
\hline \multicolumn{6}{|l|}{ Fever suppressant } \\
\hline No & 71/132 (53.79) & Ref. & 0.002 & a & - \\
\hline Yes & $75 / 102(73.53)$ & $2.39(1.37-4.17)$ & & & \\
\hline \multicolumn{6}{|c|}{ Taken anti-malarial drug } \\
\hline No & $136 / 224(60.71)$ & NA & - & a & - \\
\hline Yes & $8 / 8(100)$ & & & & \\
\hline \multicolumn{6}{|c|}{ Malaria in last 12 months } \\
\hline No & 113/193 (58.55) & Ref. & 0.011 & Ref. & 0.005 \\
\hline Yes & 33/41 (80.49) & $2.92(1.28-6.65)$ & & $3.65(1.48-9.03)$ & \\
\hline \multicolumn{6}{|l|}{ Study sites } \\
\hline Rourkela & $44 / 76(57.89)$ & Ref. & & a & - \\
\hline Chennai & $53 / 66(80.30)$ & $2.96(1.39-6.33)$ & 0.018 & & \\
\hline Nadiad & 49/92 (53.26) & $0.83(0.45-1.53)$ & 0.012 & & \\
\hline
\end{tabular}

OR odds ratio

a Omitted in multivariable analysis 
candidate antigens PvAMA- 1 and PvMSP- $1_{19}$ in individuals living in three diverse eco-epidemiological regions of India, with differing prevalence of $P$. vivax and $P$. falciparum. Total IgG antibody response was determined against these two antigens in 234 individuals living in Chennai, Nadiad and Rourkela. In these diverse ecoepidemiological study site, heterogeneity between the RDT (33.3\%), microscopy (38.0\%) and PCR (48.7\%) was observed in screening of $P$. vivax parasite prevalence. Lower parasite prevalence by RDT was observed at all three sites as compared to microscopy and PCR. Higher seroprevalence or antibody titre against these two antigens was observed at Chennai and Nadiad where $P$. vivax prevalence is high as compared to Rourkela ( $P$. falciparum dominant area). Heterogeneity in seropositivity against these two antigens was observed at all three study sites and the overall seroprevalence for PvMSP- $1_{19}$ was higher (62.4\%) as compared to PvAMA-1 (40.6\%). A total of $35.9 \%$ individuals were seropositive for both the antigens and seropositivity was higher in Chennai (45.4\%) and Nadiad (41.3\%) as compared to Rourkela (21\%). Whereas, $32.9 \%$ individuals were not showing any antibody response against PvAMA-1 and PvMSP$1_{19}$ antigens, might have slower immune response which stimulated antibodies but not up to the detectable level. This study observed PvMSP- $1_{19}$ to be highly immunogenic when compared to PvAMA-1 at all three study sites and similar observation was reported in previous immune-epidemiologic study conducted in north India [26] and other countries like Brazil and Haiti [11, 31, 3739]. A genome-scale protein microarray study of seroreactivity to different $P$. vivax and $P$. falciparum antigens conducted at these three field sites and in Goa (Southwest India) also reported merozoites surface proteins as the most immunogenic antigen in $P$. vivax and reported apical membrane antigen as most immunogenic in $P$. falciparum [40, 41]. One of the possible reason for higher immunogenicity for PvMSP- $1_{19}$ could be its conserved genetic nature, which were reported in many studies [21, 42-47] and the similar observation were recorded in our field isolates collected from Chennai, Nadiad and Rourkela (unpublished data). In contrast, Pvama-1 gene found to be highly polymorphic at these three study sites (unpublished data) and also in various previous studies [48-54]. Another possible reason could be higher exposure of PvMSP- $1_{19}$ on the surface of infected RBCs until the end of the intracellular cycle [27]. Antibody response was higher in $P$. vivax infected individuals against the PvAMA-1 antigen at all three study population. Interestingly, in Rourkela, seropositivity to PvMSP- $1_{19}$ antigen was slightly higher in $P$. vivax uninfected individuals (60.4\%, PCR negative) as compared to infected individuals (53.6\%, PCR positive), suggest antibody response against the PvMSP- $1_{19}$ antigen once acquired due to cumulative exposure of the parasite over time persist for so many years [55]. In Rourkela $25 \%$ of participants had previous exposure (in 12 months) to $P$. vivax or $P$. falciparum malaria indicates earlier exposure of malaria parasite can enhance the immune response. Another possibility is mixed infections and cross-reactivity between antibodies formed towards $P$. falciparum MSP- $1_{19}[56,57]$. Chances of being seropositive to $P$. vivax MSP-1 were highly associated with being seropositive to $P$. falciparum MSP-1, described by a previous study [56] and this could be the reason of higher seropositivity against PvMSP$1_{19}$ antigen in Rourkela where P. falciparum in dominant species. However, cross reactivity between antibodies formed against recombinant PvAMA-1 and PfAMA-1 antigen is very limited [58] and that could be the reason of less seropositivity in PvAMA-1 antigens as compared to PvMSP-119 in Rourkela. A protein array-based immune surveillance study conducted in Goa, in Southwest India where $P$. vivax is dominant species reported strong IgG response towards $P$. falciparum antigens [41]. These results highlight the need to better understand antigenic cross reactivity in areas where both $P$. falciparum and $P$. vivax co-exist. In Chennai and Nadiad population antibody response against the PvMSP- $1_{19}$ antigen was higher in $P$. vivax infected individuals. Prevalence of $P$. vivax relapse infections in an area may provide protective immunity [59], however the study did not observe any relapse cases.

Antibody response was compared in children and adults from two sites Nadiad and Rourkela as Chennai had an insufficient number of children enrolled. The antibody response against PvAMA- 1 and PvMSP- $1_{19}$ antigen was significantly higher in adults as compared to children at each study site which clearly indicate age acquired immunity. The immune response increases with age due to repeated exposure of malaria parasite [60, 61]. In addition, it was noted that the antibody response against the two antigens was higher in females as compared to males in Nadiad and Rourkela though these differences were not statistically significant. The possible reason for the differences could be the females were more often infected, therefore they might show high antibody response against the antigens. This pattern was not observed in Chennai, where antibody response was higher in males as compared to females, probably due to life style and socio-economic factor when compared to Nadiad and Rourkela. Results indicate a higher antibody response in asymptomatic individuals as compared to symptomatic, though these differences were not statistically significant. Individuals living in malaria-endemic areas are able to control parasitaemia through the immune mechanism and could remain asymptomatic $[62,63]$, which could be 
the reason for increased asymptomatic malaria cases in the study population. Antibody response against PvMSP$1_{19}$ and PvAMA-1 antigens in individuals having low parasitaemia was higher (insignificant) as compared to individuals having high parasitaemia in all three study sites. This may indicate that parasitaemia cannot be correlated with antibody response and antibodies against blood-stage antigens are developed in most individuals living in malaria endemic areas [63].

Total ten factors which could be associated with seropositivity namely sex, age, malaria positivity, symptomatic and asymptomatic malaria cases, parasitaemia, fever, fever suppressants, anti-malarial drugs, past malaria infection and heterogeneous malaria transmission sites were evaluated. Four factors age, malaria positivity (by PCR), fever (within last 2 week) and past malaria infection (in last 12 months) were significantly associated with seropositivity to PvMSP-1 $1_{19}$. Whereas, only two factors age and malaria positivity (by PCR) were significantly associated with seropositivity to PvAMA-1. Seropositivity to PvMSP- $1_{19}$ and PvAMA- 1 was higher in individuals who were having fever within last 2 weeks in Chennai, Nadiad and Rourkela and were malaria positive (by PCR), which clearly indicates that individuals with malaria infection naturally produce antibodies against these two antigens at each site resulted in higher antibody response. Individuals with past history of malaria in last 12 months recalled by memory were significantly associated with seropositivity to PvMSP- $1_{19}$ as compared to individuals who were not infected by malaria in last 12 months at each site, indicates that frequent infection increase the antibody response and boosted with subsequent attack of malaria infection [18]. Fever within last 2 weeks and diverse study sites were significantly associated with seropositivity to PvAMA- 1 and PvMSP-1 19 antigens in logistic regression analysis but omitted in multivariable analysis and larger sample size may be required to observe the association. Other factors such as symptomatic and asymptomatic malaria cases, parasitaemia, fever suppressants, and anti-malarial drugs were not significantly associated with seropositivity for both the antigens at three sites. This study has few limitations; low number of study participants and the study has lower number of children at each study site as compared to adults.

Limited studies were conducted in the Asia-Pacific region to identify immune response in a geographical diverse population with different $P$. vivax endemicity [6468]. Basic understanding of antibody response against the vaccine antigens in different geographical areas is important to assess the effectiveness of malaria vaccine. This study clearly demonstrated that individuals living in three malaria endemic areas of India greatly vary in their antibody response to these two leading blood-stage vaccine antigens. PvMSP- $1_{19}$ is highly immunogenic and recognized more strongly in different geographical population. The varied antibody response observed between two antigens in the present study could be human genetic background, antigenic polymorphism, structural differences in the antigens, differential responsiveness and differences in $P$. vivax transmission in the study sites, which needs further investigation.

\section{Conclusion}

In conclusion, it is evident that $P$. vivax $\mathrm{MSP}-1_{19}$ is highly immunogenic during natural infection in individuals living in three geographically diverse malaria endemic regions of India. There is heterogeneity in antibody response to PvMSP-1 19 and PvAMA-1 antigen among three populations. Results obtained here have implications for understanding human immunity to malaria antigens in different populations and could be helpful in malaria elimination programme and vaccine development.

\section{Abbreviations}

MSP-1 1 : merozoite surface protein-1 1 ; PVAMA-1: apical membrane antigen-1; LM: light microscopy; PCR: polymerase chain reaction; ELISA: enzyme linked immunosorbent assay; OD: optical density; OR: odds ratio; $\mathrm{Cl}$ : confidence interval.

\section{Acknowledgements}

The authors would like to thank Dr. Chris Drakeley and Dr. Kevin Tetteh (London School of Hygiene and Tropical Medicine, London, UK) for providing recombinant proteins PVAMA-1 and PVMSP-1 19 . We are grateful to the staff of the National Institute of Malaria Research in Delhi and at three NIMR field unit. We thank Dr. Manish Manish and Dr. Surendra Kumar Prajapati for their scientific suggestions. We are grateful to all the study participants. This manuscript bears the National Institute of Malaria Research publication screening committee approval number 07/2019.

\section{Authors' contributions}

OPS, JMC, PNR, VP, PKM, AE, HCS, SKS designed the study. OPS, JMC, PKM contributed reagents/materials/analysis tools. SK performed the experiments. SK, CPY, PKM analysed and interpreted data. SK wrote the first draft. SK, OPS, PKM, CYP, SS reviewed and edited the manuscript. All authors read and approved the final manuscript.

\section{Funding}

The research reported in this publication was supported by the National Institute of Allergy and Infectious Diseases of the National Institutes of Health under Award Number U19AI089676 as part of the International Centers for Excellence in Malaria Research. The content is solely the responsibility of the authors and does not necessarily represent the official views of the National Institutes of Health.

\section{Availability of data and materials}

All data generated or analysed during this study are included in this published article.

\section{Ethics approval and consent to participate}

This study was conducted as a part of the NIH funded project "Centre for the study of complex Malaria in India" which was approved by the Institutional

Ethics Committee of ICMR-NIMR in New Delhi, India and New York University 
Institutional Review Board. Clinical samples were collected after informed consent was obtained from all participants. Consent for children (under 18 years) was obtained from their parents or guardian.

\section{Consent for publication}

Not applicable.

\section{Competing interests}

The authors declare that they have no competing interests.

\section{Author details}

${ }^{1}$ ICMR-National Institute of Malaria Research, Sector 8, Dwarka, New Delhi, India. ${ }^{2}$ Department of Biotechnology, Kumaun University, Nainital, Uttarakhand, India. ${ }^{3}$ Department of Biology, Center for Genomics and Systems Biology, New York University, New York, USA. ${ }^{4}$ National Institute of Malaria Research Field Unit, Indian Council of Medical Research, National Institute of Epidemiology Campus, Ayapakkam, Chennai, Tamil Nadu, India. ${ }^{5}$ National Institute of Malaria Research Field Unit, Civil Hospital, Nadiad, Gujarat, India. ${ }^{6}$ Jigyansha, International Center of Excellence for Malaria Research, Sector 1, Rourkela, Odisha, India.

Received: 18 September 2019 Accepted: 8 December 2019

Published online: 16 December 2019

\section{References}

1. WHO Global Malaria Programme. World malaria report 2018. Geneva: World Health Organization; 2018. http://www.who.int/malaria/publicatio ns/world-malaria-report-2018/en/.

2. WHO Global Malaria Programme. World malaria report 2017. Geneva: World Health Organization; 2017. http://www.who.int/malaria/publicatio ns/world-malaria-report-2017/en/.

3. National Vector Borne Disease Control Programme, Directorate General of Health Services, Ministry of Health and Family Welfare \& Government of India. National strategic plan malaria elimination in India 2017-2022. https://www.nvbdcp.gov.in/index.php.

4. Thera MA, Plowe CV. Vaccines for malaria: how close are we? Annu Rev Med. 2012;63:345-57.

5. Fonseca JA, Cabrera-Mora M, Singh B, Oliveira-Ferreira J, da Costa LimaJunior J, Calvo-Calle JM, et al. A chimeric protein-based malaria vaccine candidate induces robust $\mathrm{T}$ cell responses against Plasmodium vivax MSP119. Sci Rep. 2016;6:34527.

6. Kocken $\mathrm{CH}$, Dubbeld MA, Van Der Wel A, Pronk JT, Waters AP, Langermans JA, et al. High-level expression of Plasmodium vivax apical membrane antigen 1 (AMA-1) in Pichia pastoris: strong immunogenicity in Macaca mulatta immunized with P. vivax AMA-1 and adjuvant SBAS2. Infect Immun. 1999;67:43-9.

7. Wilson DW, Fowkes FJ, Gilson PR, Elliott SR, Tavul L, Michon P, et al. Quantifying the importance of MSP1-19 as a target of growth-inhibitory and protective antibodies against Plasmodium falciparum in humans. PLoS ONE. 2011;6:e27705.

8. Cowman AF, Berry D, Baum J. The cellular and molecular basis for malaria parasite invasion of the human red blood cell. J Cell Biol. 2012;198:961-71.

9. Arnot DE, Cavanagh DR, Remarque EJ, Creasey AM, Sowa MP, Morgan WD, et al. Comparative testing of six antigen-based malaria vaccine candidates directed toward merozoite-stage Plasmodium falciparum. Clin Vaccine Immunol. 2008;15:1345-55.

10. Rocha MV, Francoso KS, Lima LC, Camargo TM, Machado RLD, Costa FTM, et al. Generation, characterization and immunogenicity of a novel chimeric recombinant protein based on Plasmodium vivax AMA-1 and MSP119. Vaccine. 2017;35:2463-72.

11. Cassiano GC, Furini AA, Capobianco MP, Storti-Melo LM, Cunha MG, Kano FS, et al. Polymorphisms in B Cell co-stimulatory genes are associated with igg antibody responses against blood-stage proteins of Plasmodium vivax. PLOS ONE. 2016;11:e0149581.

12. Blackman MJ, Heidrich HG, Donachie S, McBride JS, Holder AA. A single fragment of a malaria merozoite surface protein remains on the parasite during red cell invasion and is the target of invasion-inhibiting antibodies. J Exp Med. 1990;172:379-82.
13. Holder AA, Blackman MJ, Burghaus PA, Chappel JA, Ling IT, McCallumDeighton N, et al. A malaria merozoite surface protein (MSP1)-structure, processing and function. Mem Inst Oswaldo Cruz. 1992;87(Suppl 3):37-42.

14. Gerold P, Schofield L, Blackman MJ, Holder AA, Schwarz RT. Structural analysis of the glycosyl-phosphatidylinositol membrane anchor of the merozoite surface proteins- 1 and -2 of Plasmodium falciparum. Mol Biochem Parasitol. 1996;75:131-43.

15. Pires CV, Alves JRS, Lima BAS, Paula RB, Costa HL, Torres LM, et al. Blood-stage Plasmodium vivax antibody dynamics in a low transmission setting: a nine year follow-up study in the Amazon region. PLoS ONE. 2018;13:e0207244.

16. Hodder AN, Crewther PE, Anders RF. Specificity of the protective antibody response to apical membrane antigen 1. Infect Immun. 2001;69:3286-94.

17. Dutta S, Haynes JD, Barbosa A, Ware LA, Snavely JD, Moch JK, et al. Mode of action of invasion-inhibitory antibodies directed against apical membrane antigen 1 of Plasmodium falciparum. Infect Immun. 2005:73:2116-22.

18. Doolan DL, Dobano C, Baird JK. Acquired immunity to malaria. Clin Microbiol Rev. 2009:22:13-36.

19. Dodoo D, Aikins A, Kusi KA, Lamptey H, Remarque E, Milligan P, et al. Cohort study of the association of antibody levels to AMA1, MSP119, MSP3 and GLURP with protection from clinical malaria in Ghanaian children. Malar J. 2008;7:142.

20. Idris ZM, Chan CW, Kongere J, Hall T, Logedi J, Gitaka J, et al. Naturally acquired antibody response to Plasmodium falciparum describes heterogeneity in transmission on islands in Lake Victoria. Sci Rep. 2017;7:9123.

21. Soares IS, Oliveira SG, Souza JM, Rodrigues MM. Antibody response to the $\mathrm{N}$ and $\mathrm{C}$-terminal regions of the Plasmodium vivax Merozoite Surface Protein 1 in individuals living in an area of exclusive transmission of $P$. vivax malaria in the north of Brazil. Acta Trop. 1999;72:13-24.

22. Takala SL, Plowe CV. Genetic diversity and malaria vaccine design, testing and efficacy: preventing and overcoming 'vaccine resistant malaria'. Parasite Immunol. 2009;31:560-73.

23. Ling IT, Ogun SA, Holder AA. Immunization against malaria with a recombinant protein. Parasite Immunol. 1994;16:63-7.

24. Daly TM, Long CA. Humoral response to a carboxyl-terminal region of the merozoite surface protein-1 plays a predominant role in controlling blood-stage infection in rodent malaria. J Immunol. 1995;155:236-43.

25. Holder AA, Guevara Patino JA, Uthaipibull C, Syed SE, Ling IT, Scott-Finnigan T, et al. Merozoite surface protein 1, immune evasion, and vaccines against asexual blood stage malaria. Parassitologia. 1999;41:409-14

26. Seth RK, Bhat AA, Rao DN, Biswas S. Acquired immune response to defined Plasmodium vivax antigens in individuals residing in northern India. Microbes Infect. 2010;12:199-206.

27. Moss DK, Remarque EJ, Faber BW, Cavanagh DR, Arnot DE, Thomas AW, et al. Plasmodium falciparum 19-kilodalton merozoite surface protein 1 (MSP1)-specific antibodies that interfere with parasite growth in vitro can inhibit MSP1 processing, merozoite invasion, and intracellular parasite development. Infect Immun. 2012;80:1280-7.

28. Das A, Anvikar AR, Cator LJ, Dhiman RC, Eapen A, Mishra N, et al. Malaria in India: the center for the study of complex malaria in India. Acta Trop. 2012;121:267-73.

29. van Eijk AM, Ramanathapuram L, Sutton PL, Peddy N, Choubey S, Mohanty S, et al. The use of mosquito repellents at three sites in India with declining malaria transmission: surveys in the community and clinic. Parasit Vectors. 2016;9:418.

30. Conn JE, Norris DE, Donnelly MJ, Beebe NW, Burkot TR, Coulibaly MB, et al. Entomological monitoring and evaluation: diverse transmission settings of ICEMR projects will require local and regional malaria elimination strategies. Am J Trop Med Hyg. 2015;93:28-41.

31. Moss WJ, Dorsey G, Mueller I, Laufer MK, Krogstad DJ, Vinetz JM, et al. Malaria epidemiology and control within the International Centers of Excellence for Malaria Research. Am J Trop Med Hyg. 2015:93:5-15.

32. Rubio JM, Benito A, Roche J, Berzosa PJ, Garcia ML, Mico M, et al. Seminested, multiplex polymerase chain reaction for detection of human malaria parasites and evidence of Plasmodium vivax infection in Equatorial Guinea. Am J Trop Med Hyg. 1999;60:183-7.

33. Drakeley CJ, Corran PH, Coleman PG, Tongren JE, McDonald SL, Carneiro I, et al. Estimating medium- and long-term trends in malaria transmission 
by using serological markers of malaria exposure. Proc Natl Acad Sci USA. 2005;102:5108-13.

34. Burghaus PA, Holder AA. Expression of the 19-kilodalton carboxy-terminal fragment of the Plasmodium falciparum merozoite surface protein-1 in Escherichia coli as a correctly folded protein. Mol Biochem Parasitol. 1994;64:165-9.

35. Franks $\mathrm{S}$, Baton $\mathrm{L}$, Tetteh $\mathrm{K}$, Tongren E, Dewin D, Akanmori BD, et al. Genetic diversity and antigenic polymorphism in Plasmodium falciparum: extensive serological cross-reactivity between allelic variants of merozoite surface protein 2. Infect Immun. 2003;71:3485-95.

36. Corran PH, Cook J, Lynch C, Leendertse H, Manjurano A, Griffin J, et al. Dried blood spots as a source of anti-malarial antibodies for epidemiological studies. Malar J. 2008;7:195.

37. Weppelmann TA, von Fricken ME, Lam B, Telisma T, Existe A, Lemoine JF, et al. Sparse serological evidence of Plasmodium vivax transmission in the Ouest and Sud-Est departments of Haiti. Acta Trop. 2016;162:27-34.

38. Soares IS, Levitus G, Souza JM, Del Portillo HA, Rodrigues MM. Acquired immune responses to the $\mathrm{N}$ - and $\mathrm{C}$-terminal regions of Plasmodium vivax merozoite surface protein 1 in individuals exposed to malaria. Infect Immun. 1997:65:1606-14.

39. Barbedo MB, Ricci R, Jimenez MC, Cunha MG, Yazdani SS, Chitnis CE, et al. Comparative recognition by human IgG antibodies of recombinant proteins representing three asexual erythrocytic stage vaccine candidates of Plasmodium vivax. Mem Inst Oswaldo Cruz. 2007;102:335-9.

40. Uplekar S, Rao PN, Ramanathapuram L, Awasthi V, Verma K, Sutton P, et al. Characterizing antibody responses to Plasmodium vivax and Plasmodium falciparum antigens in India using genome-scale protein microarrays. PLoS Negl Trop Dis. 2017;11:e0005323.

41. Venkatesh A, Jain A, Davies H, Periera L, Maki JN, Gomes E, et al. Hospitalderived antibody profiles of malaria patients in Southwest India. Malar J. 2019;18:138.

42. O'Donnell RA, Saul A, Cowman AF, Crabb BS. Functional conservation of the malaria vaccine antigen MSP-119 across distantly related Plasmodium species. Nat Med. 2000;6:91-5.

43. Putaporntip C, Jongwutiwes S, Sakihama N, Ferreira MU, Kho WG, Kaneko $A$, et al. Mosaic organization and heterogeneity in frequency of allelic recombination of the Plasmodium vivax merozoite surface protein-1 locus. Proc Natl Acad Sci USA. 2002;99:16348-53.

44. Pacheco MA, Poe AC, Collins WE, Lal AA, Tanabe K, Kariuki SK, et al. A comparative study of the genetic diversity of the $42 \mathrm{kDa}$ fragment of the merozoite surface protein 1 in Plasmodium falciparum and P. vivax. Infect Genet Evol. 2007;7:180-7.

45. del Portillo HA, Longacre S, Khouri E, David PH. Primary structure of the merozoite surface antigen 1 of Plasmodium vivax reveals sequences conserved between different Plasmodium species. Proc Natl Acad Sci USA. 1991:88:4030-4

46. Soares IS, Barnwell JW, Ferreira MU, Da Cunha MG, Laurino JP, Castilho $\mathrm{BA}$, et al. A Plasmodium vivax vaccine candidate displays limited allele polymorphism, which does not restrict recognition by antibodies. Mol Med. 1999;5:459-70.

47. Thakur A, Alam MT, Sharma YD. Genetic diversity in the C-terminal $42 \mathrm{kDa}$ region of merozoite surface protein-1 of Plasmodium vivax (PvMSP-1 (42)) among Indian isolates. Acta Trop. 2008;108:58-63.

48. Arnott A, Mueller I, Ramsland PA, Siba PM, Reeder JC, Barry AE. Global population structure of the genes encoding the malaria vaccine candidate, Plasmodium vivax apical membrane antigen 1 (PvAMA1). PLoS Negl Trop Dis. 2013;7:e2506.

49. Figtree M, Pasay CJ, Slade R, Cheng Q, Cloonan N, Walker J, et al. Plasmodium vivax synonymous substitution frequencies, evolution and population structure deduced from diversity in AMA 1 and MSP 1 genes. Mol Biochem Parasitol. 2000;108:53-66.

50. Heidari A, Keshavarz H, Hajjaran H, Ebrahimi SM, Kabir K, Naseri MH. Genetic variation and selection of domain I of the Plasmodium vivax apical membrane antigen-1 (AMA-1) gene in clinical isolates from Iran. Iran 」 Parasitol. 2013;8:536-44.

51. Rajesh V, Elamaran M, Vidya S, Gowrishankar M, Kochar D, Das A. Plasmodium vivax: genetic diversity of the apical membrane antigen-1 (AMA-1) in isolates from India. Exp Parasitol. 2007;116:252-6.
52. Thakur A, Alam MT, Bora H, Kaur P, Sharma YD. Plasmodium vivax: sequence polymorphism and effect of natural selection at apical membrane antigen 1 (PvAMA1) among Indian population. Gene. 2008:419:35-42.

53. Zakeri S, Sadeghi H, Mehrizi AA, Djadid ND. Population genetic structure and polymorphism analysis of gene encoding apical membrane antigen-1 (AMA-1) of Iranian Plasmodium vivax wild isolates. Acta Trop. 2013;126:269-79.

54. Zhu X, Zhao P, Wang S, Liu F, Liu J, Wang J, et al. Analysis of Pvama1 genes from China-Myanmar border reveals little regional genetic differentiation of Plasmodium vivax populations. Parasit Vectors. 2016;9:614.

55. Okell LC, Bousema T, Griffin JT, Ouedraogo AL, Ghani AC, Drakeley CJ. Factors determining the occurrence of submicroscopic malaria infections and their relevance for control. Nat Commun. 2012;3:1237.

56. von Fricken ME, Weppelmann TA, Lam B, Eaton WT, Schick L, Masse R, et al. Age-specific malaria seroprevalence rates: a cross-sectional analysis of malaria transmission in the Ouest and Sud-Est departments of Haiti. Malar J. 2014;13:361.

57. Akpogheneta OJ, Dunyo S, Pinder M, Conway DJ. Boosting antibody responses to Plasmodium falciparum merozoite antigens in children with highly seasonal exposure to infection. Parasite Immunol. 2010;32:296-304.

58. Igonet $\mathrm{S}$, Vulliez-Le Normand B, Faure G, Riottot MM, Kocken CH, Thomas AW, et al. Cross-reactivity studies of an anti-Plasmodium vivax apical membrane antigen 1 monoclonal antibody: binding and structural characterisation. J Mol Biol. 2007;366:1523-37.

59. White NJ. Determinants of relapse periodicity in Plasmodium vivax malaria. Malar J. 2011;10:297.

60. Griffin JT, Hollingsworth TD, Reyburn H, Drakeley CJ, Riley EM, Ghani AC. Gradual acquisition of immunity to severe malaria with increasing exposure. Proc Biol Sci. 2015;282:20142657.

61. Stanisic DI, Fowkes FJ, Koinari M, Javati S, Lin E, Kiniboro B, et al. Acquisition of antibodies against Plasmodium falciparum merozoites and malaria immunity in young children and the influence of age, force of infection, and magnitude of response. Infect Immun. 2015;83:646-60.

62. Nwagwu M, Anumudu CA, Sodeinde O, Ologunde CA, Obi TU, Wirtz RA, et al. Identification of a subpopulation of immune Nigerian adult volunteers by antibodies to the circumsporozoite protein of Plasmodium falciparum. Am J Trop Med Hyg. 1998;58:684-92.

63. Biswas S, Seth RK, Tyagi PK, Sharma SK, Dash AP. Naturally acquired immunity and reduced susceptibility to falciparum malaria in two subpopulations of endemic eastern India. Scand J Immunol. 2008;67:177-84.

64. Cutts JC, Powell R, Agius PA, Beeson JG, Simpson JA, Fowkes FJ. Immunological markers of Plasmodium vivax exposure and immunity: a systematic review and meta-analysis. BMC Med. 2014;12:150.

65. Guerra CA, Howes RE, Patil AP, Gething PW, Van Boeckel TP, Temperley WH, et al. The international limits and population at risk of Plasmodium vivax transmission in 2009. PLoS Negl Trop Dis. 2010;4:e774.

66. Cook J, Speybroeck N, Sochanta T, Somony H, Sokny M, Claes F, et al. Sero-epidemiological evaluation of changes in Plasmodium falciparum and Plasmodium vivax transmission patterns over the rainy season in Cambodia. Malar J. 2012;11:86.

67. Ak M, Jones TR, Charoenvit Y, Kumar S, Kaslow DC, Maris D, et al. Humoral immune responses against Plasmodium vivax MSP1 in humans living in a malaria endemic area in Flores, Indonesia. Southeast Asian J Trop Med Public Health. 1998;29:685-91.

68. Cole-Tobian JL, Michon P, Biasor M, Richards JS, Beeson JG, Mueller I, et al. Strain-specific duffy binding protein antibodies correlate with protection against infection with homologous compared to heterologous Plasmodium vivax strains in Papua New Guinean children. Infect Immun. 2009;77:4009-17.

\section{Publisher's Note}

Springer Nature remains neutral with regard to jurisdictional claims in published maps and institutional affiliations. 